\title{
Cognitive Assessment of Elderly Inpatients: A Clinical Audit
}

\author{
Elizabeth Shermon Lucy O. Vernon Adrian J. McGrath \\ University of Birmingham, Edgbaston Campus, Birmingham, UK
}

\author{
Key Words \\ Cognitive assessment · Elderly · Dementia - Dementia screening · Service delivery · Geriatrics . \\ Education
}

\begin{abstract}
Background: Comprehensive geriatric assessment including cognitive assessment results in better outcomes and quality of life through facilitating access to support and further care. The National Audit of Dementia Care revealed too few patients were being assessed for cognition and therefore failing to receive adequate care. Methods: This was a retrospective clinical audit in a district general hospital with systematic sampling of the clinical records of 50 inpatients on an elderly care ward. A descriptive analysis of the results was performed. Results: Despite guidance that cognitive assessment should be performed on admission, this was only documented in $22 \%$ of the medical notes. However, this rate improved to $56 \%$ by discharge. The most commonly used tool was the Abbreviated Mental Test (AMT) 10. Assessment completion was independent of gender or social support, but only patients aged over 75 years were assessed. Of those, $75 \%$ had some level of cognitive impairment and $36.8 \%$ received a new or suspected diagnosis of dementia. Discussion: Cognitive assessment rates continue to be low. Our findings support the need for increased education regarding the importance and benefits of assessment as well as how to complete and document the assessment correctly. Conclusion: Cognitive assessment rates need to be further improved to promote better outcomes for patients with dementia.

(C) 2015 S. Karger AG, Basel
\end{abstract}

\section{Introduction}

Previous research has shown that comprehensive geriatric assessment including cognitive assessment results in better outcomes and improved quality of life for elderly hospital inpatients [1]. By detecting a cognitive impairment, further investigations can be 
completed, which may then result in a diagnosis. This process enables problems to be identified and an integrated and coordinated plan for treatment and follow-up to be initiated, which may help to maximise recovery, function and quality of life [1]. The National Audit of Dementia Care revealed that too few patients were being assessed for cognitive function and were therefore failing to receive adequate care. Consequently, recommendations were made to improve the implementation of cognitive assessment, which formed the foundation for this clinical audit [2]. During our clinical practice, we noticed that cognitive assessment was being poorly performed, and we aimed to investigate this further and compare local practice at a district general hospital to national standards and guidelines.

\section{Materials and Methods}

Systematic sampling was used to select 50 clinical records from patients admitted to the elderly care ward at a district general hospital in the West Midlands, UK, in September 2013. Patients were admitted to this ward if it was considered that their care needs would be best met under the management of geriatricians. A cross-sectional approach was used with any current inpatient on this ward at the point of data collection being eligible for inclusion in the study. In order to collect the data, the written clinical records of patients in odd-numbered bed spaces were assessed. Since allocation to any given bed space was independent of patient characteristics, this should have produced a relatively random selection of records. The written records were then assessed for documentation of completed cognitive assessment by various healthcare professionals in addition to patient demographics and characteristics. The results were then analysed using a descriptive approach in order to summarise the characteristics of the population group with quantitative data including percentages and averages.

\section{Results (table 1)}

Despite guidelines suggesting that cognitive assessment should be performed on admission, with good practice dictating that the clerking doctor should complete this as part of the patient's initial assessment, we found that this was only documented in $22 \%$ of the medical notes. Where assessment is not completed on admission or in cases where further assessment is clinically indicated, it is appropriate for doctors on the ward to complete further tests. This is reflected in our finding that assessment rates improved to $56 \%$ by discharge (fig. 1). Although these figures initially appear disappointing, they are higher than the level reported in the National Audit of Dementia Care, which found mental status was only assessed in $50 \%$ of patients by discharge. We considered the findings of the National Audit of Dementia Care to be a minimum standard to achieve, and our results indicate that local performance was better than the expected standard.

The most commonly used assessment tool was the Abbreviated Mental Test (AMT) 10, which was used in $85.7 \%$ of the cases. This test is a 10 -point questionnaire which assesses orientation to person, place and time, attention and calculation, recall and repetition. The Mini-Mental State Examination (MMSE) was used in a further $10.7 \%$ of assessments. In addition to the skills above, this 30-point questionnaire also assesses the ability to perform complex tasks [3]. The remainder of the assessments used the AMT4. Of the tests completed, $67.9 \%$ were done by doctors, $28.6 \%$ by nurses and $3.6 \%$ by medical students.

Assessment completion was largely independent of patient gender, with assessment being completed in $52.6 \%$ of males and $58.1 \%$ of females. There was also no trend between the patient's level of independence and whether cognitive assessment was performed, with 


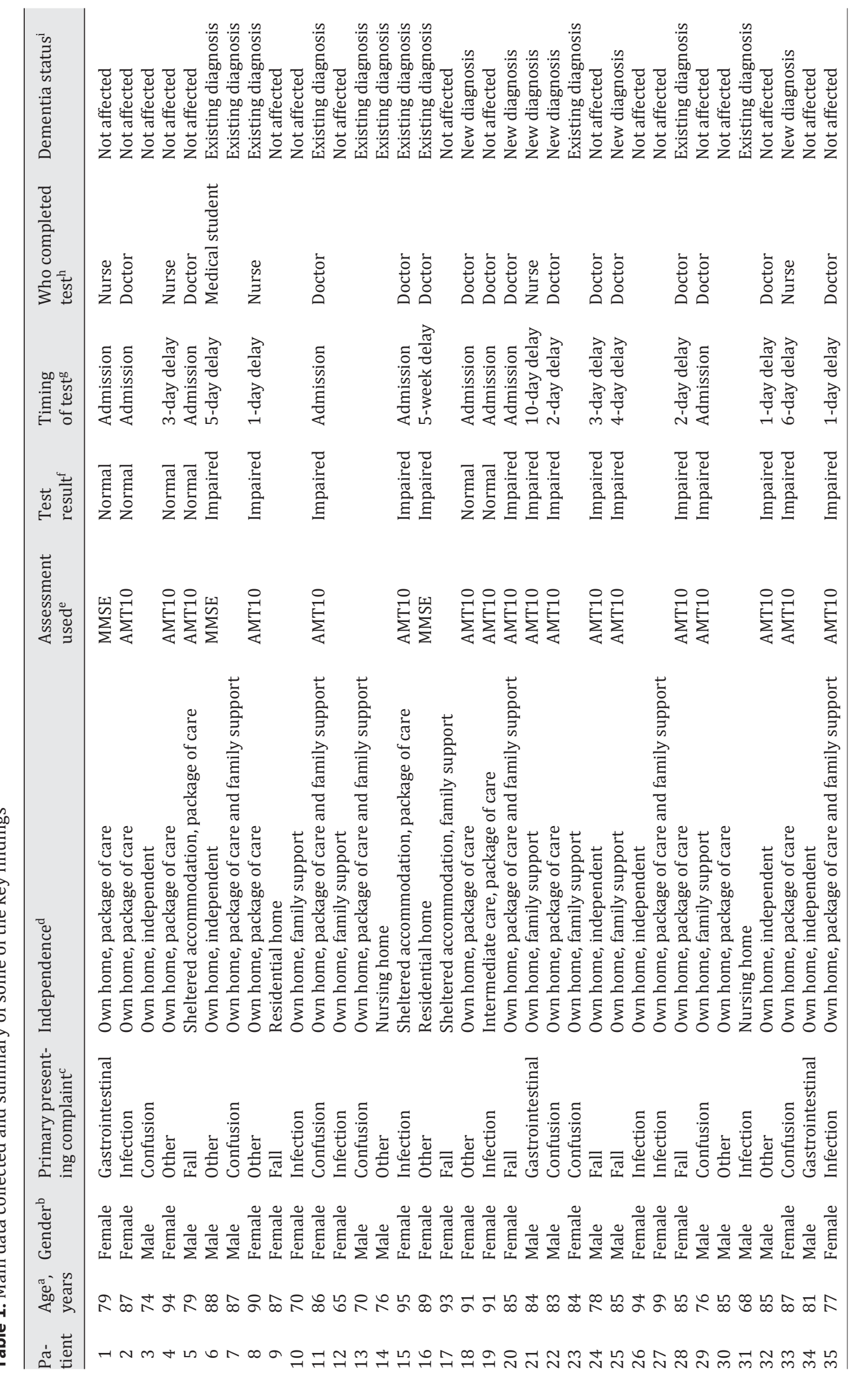




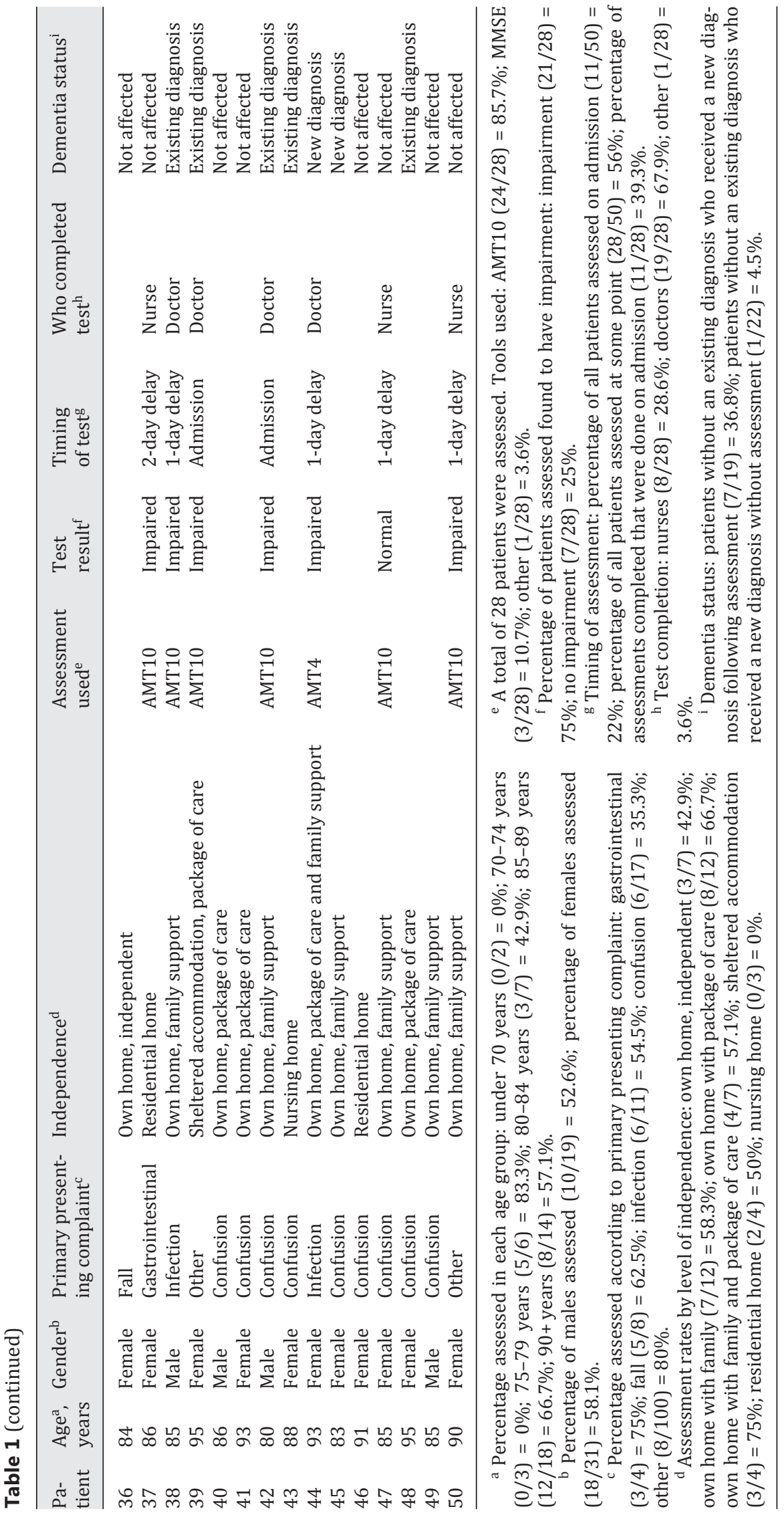


Fig. 1. Rates and timing of cognitive assessment. Total number of patients $=50$.

Fig. 2. Rates of assessment by age.
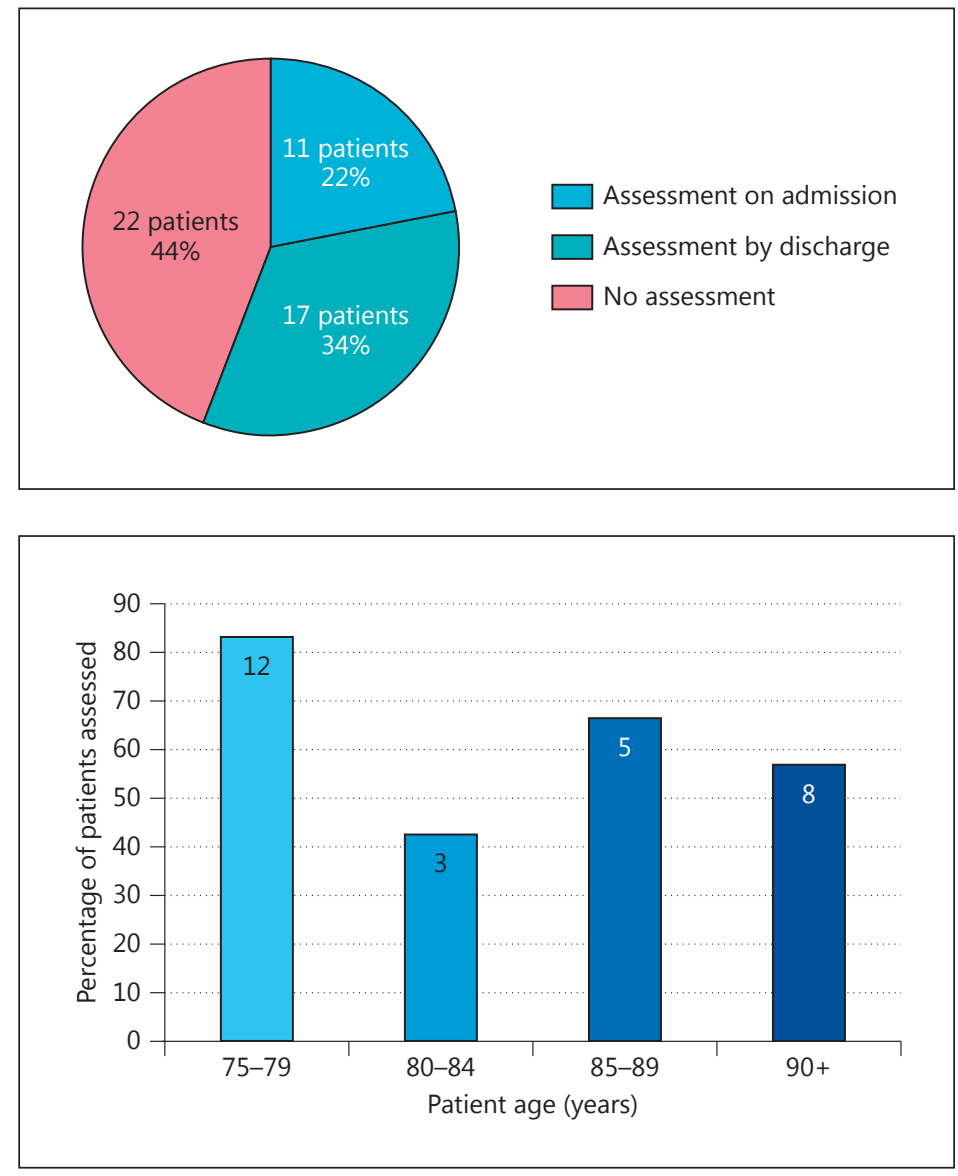

similar frequencies amongst patients with different care needs. We assessed the level of independence in accordance to the patients' place of residence, package of care and level of family support prior to admission as indicated in their notes. Assessment was only performed in patients over the age of 75 years, with varying frequency between age subgroups. The highest rate of completion (83\%) was seen in patients aged 75-79 years. In the 80-84 age group, $42.9 \%$ of the patients were assessed; this rate increased to $66.7 \%$ in those aged $85-89$ years, before it again fell to $57.1 \%$ in patients aged 90 years and above (fig. 2). There is no clear explanation for this variation.

There was no clear relationship between completion of cognitive assessment and reason for hospital admission; however, it was noted that assessment rates were particularly poor in those documented to be confused on admission, with only $35.3 \%$ of these patients being assessed. The term confusion relates to the information provided by the clerking doctor either as part of the presenting complaint or as an examination finding during the initial assessment.

Interestingly, of the patients assessed, $75 \%$ were found to have some level of cognitive impairment. We defined cognitive impairment as a score of $\leq 7$ on the AMT10 assessment, a score of $<24$ on the MMSE or a score of $<4$ on the AMT4. Furthermore, $36.8 \%$ of the assessed patients went on to receive a new suspected or confirmed diagnosis of dementia as documented in the medical notes by an appropriately qualified specialist after relevant investigations had been performed [3]. In contrast, of those patients who failed to receive cognitive assessment, only $4.5 \%$ were diagnosed with dementia, highlighting the increased rates of detection when assessment is completed. It is difficult to follow in the patient notes how this 
Shermon et al.: Cognitive Assessment of Elderly Inpatients: A Clinical Audit

diagnosis was reached in the absence of a record of cognitive assessment. It is presumed that either formal cognitive assessment was completed but not documented in the medical notes or the diagnosis was made by a geriatrician on the basis of the patients' clinical presentation.

\section{Discussion}

Our results show that, despite reaching above the expected standard, completion of cognitive assessment is poor, especially on admission, where only $22 \%$ of the patients were assessed. One explanation for this finding could be the attitudes of the doctors responsible for clerking and admitting patients. Despite a clear section on the clerking form presenting the AMT10 in a tick-box format, we found that this section of the form was frequently ignored or inadequately completed. There are many proposed reasons why the AMT10 may not be completed, including lack of privacy to conduct the test, time constraints, perceived lack of expertise, poor attitudes towards cognitive assessment and lack of understanding the importance of such testing [4]. Such opinions became apparent during data collection, where several doctors openly admitted that they frequently failed to complete that section of the clerking form. However, the AMT10 was the most commonly used assessment tool, reflecting its presence on the clerking form.

Our audit found that only patients aged 75 years and above were assessed for cognitive function. However, research by the Alzheimer's Society has shown that mild cognitive impairment is present in 10-20\% of adults over the age of 65 years [5]. In mild cognitive impairment, there may be a measurable decline in memory and thinking skills, which may be noted by friends and family [5]. Furthermore, patients who develop mild cognitive impairment are at an increased risk of developing dementia in the future, so this condition needs to be detected and monitored. As the symptoms are often more subtle in younger patients, the need for formal assessment is greater, so that further management can be implemented [5]. Since earlier detection of cognitive impairment and dementia results in better outcomes, including improved quality of life, the ability to return to independent living following hospital admission, reduction in number and length of hospital admissions and better utilisation of health and social resources, it is clearly imperative that clinicians assess patients from a younger age [6]. This is reflected in the British Geriatric Society guidelines, which are based around the concept of improving outcomes for patients.

In addition, we discovered that assessment rates were particularly poor amongst patients presenting with confusion. This seems counterintuitive since it makes monitoring of their condition difficult if no record of their cognitive function on admission is made. Assessing cognitive function in patients presenting with confusion is particularly important since this may be a symptom of delirium and evidence has shown that there is a higher incidence of dementia in these patients. It can also be difficult to distinguish between delirium and dementia, and cognitive assessment can be used as an important tool to differentiate between them [7]. Furthermore, if serial assessments are not made throughout the patients' admission, it is impossible to detect any long-standing milder impairment that may be masked by their acute confusional state.

Finally, the findings of our audit support the use of screening tools for detecting cognitive impairment, with higher rates of suspected or confirmed diagnoses of dementia in those patients assessed.

There were a number of limitations in our study, such as the small cohort size. Data were collected from only one ward, in one hospital, at one point of time, and, therefore, it is hard to apply the results more widely across the UK. In addition, since our methodology was different to that used in the National Audit of Dementia Care, direct comparisons cannot be made. 


\section{Recommendations}

In order to improve attitudes towards cognitive assessment, education on its importance and the benefits of earlier detection should be highlighted to students during medical school training. In doing so, it is hoped that junior doctors will start work with a full awareness of the merits of cognitive assessment and a better appreciation of the improved prognosis associated with earlier detection. Subsequently, attitudes towards completing cognitive assessment should be improved.

In addition, the need to complete all sections of the clerking form when admitting patients should be emphasised to junior doctors during their induction. More senior doctors should also be reminded of the need to monitor and encourage the use of these tools to improve patient care and perform the tests themselves when they are absent or inadequately completed in patient notes.

It should also be highlighted to all healthcare professionals that the need to perform cognitive assessment should be considered in all patients, regardless of age, but especially in those aged 65 years and above.

\section{Conclusions}

Cognitive assessment continues to be poorly performed in elderly patients. Although higher cognitive assessment rates than those found by the National Audit of Dementia Care were achieved in this audit, there is still room for improvement. Since performing cognitive assessment leads to earlier detection of and better outcomes in dementia, doctors should be encouraged to use these tools to improve patient care. It may be possible to improve cognitive assessment rates through better education and training for medical students and junior doctors.

\section{Disclosure Statement}

The authors have no conflicts of interest to declare.

\section{References}

1 Martin F: Comprehensive assessment of the frail older patient. British Geriatrics Society. 2010. http://www. bgs.org.uk/index.php/topresources/publicationfind/goodpractice/195-gpgcgassessment (accessed November 10, 2013).

2 Royal College of Psychiatrists: National Audit of Dementia care in general hospitals 2012-13: second round audit report and update. 2013. http://www.rcpsych.ac.uk/pdf/NAD\%20NATIONAL\%20REPORT\%202013. pdf (accessed November 10, 2013).

3 Alzheimer's Society: Helping you to assess cognition: a practical toolkit for clinicians. 2013. www.alzheimers. org.uk/site/scripts/download.php?fileID=1661 (accessed January 10, 2015).

4 Kennelly SP, Morley D, Coughlan T, Collins R, Rochford M, O’Neill D: Knowledge, skills and attitudes of doctors towards assessing cognition in older patients in the emergency department. Postgrad Med J 2013;89:137141.

5 Alzheimer's Association: Mild cognitive impairment (MCI). 2012. http://www.alz.org/dementia/downloads/ topicsheet_mci.pdf (accessed February 23, 2014).

6 Royal College of Psychiatrists: Who cares wins. Improving the outcome for older people admitted to the general hospital: guidelines for the development of Liaison Mental Health Services for older people. 2005. (accessed January 10, 2015).

7 National Institute of Clinical Excellence: Delirium: diagnosis, prevention and management (NICE clinical guideline 103). 2010. http://www.nice.org.uk/nicemedia/live/13060/49909/49909.pdf (accessed March $16,2014)$. 